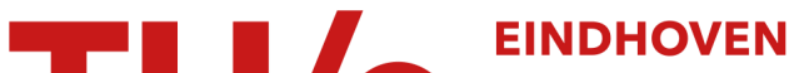 UNIVERSITY OF TECHNOLOGY
}

\section{From Lie algebras of vector fields to algebraic group actions}

Citation for published version (APA):

Cohen, A. M., \& Draisma, J. (2003). From Lie algebras of vector fields to algebraic group actions.

Transformation Groups, 8(1), 51-68. https://doi.org/10.1007/s00031-003-1210-3

DOI:

10.1007/s00031-003-1210-3

Document status and date:

Published: 01/01/2003

\section{Document Version:}

Publisher's PDF, also known as Version of Record (includes final page, issue and volume numbers)

\section{Please check the document version of this publication:}

- A submitted manuscript is the version of the article upon submission and before peer-review. There can be important differences between the submitted version and the official published version of record. People interested in the research are advised to contact the author for the final version of the publication, or visit the $\mathrm{DOI}$ to the publisher's website.

- The final author version and the galley proof are versions of the publication after peer review.

- The final published version features the final layout of the paper including the volume, issue and page numbers.

Link to publication

\section{General rights}

Copyright and moral rights for the publications made accessible in the public portal are retained by the authors and/or other copyright owners and it is a condition of accessing publications that users recognise and abide by the legal requirements associated with these rights.

- Users may download and print one copy of any publication from the public portal for the purpose of private study or research.

- You may not further distribute the material or use it for any profit-making activity or commercial gain

- You may freely distribute the URL identifying the publication in the public portal.

If the publication is distributed under the terms of Article $25 \mathrm{fa}$ of the Dutch Copyright Act, indicated by the "Taverne" license above, please follow below link for the End User Agreement:

www.tue.nl/taverne

Take down policy

If you believe that this document breaches copyright please contact us at:

openaccess@tue.nl

providing details and we will investigate your claim. 


\title{
FROM LIE ALGEBRAS OF VECTOR FIELDS TO ALGEBRAIC GROUP ACTIONS
}

\section{ARJEH M. COHEN}

Department of Mathematics and Computing Science

Technische Universiteit Eindhoven P.O. Box 513, $5600 \mathrm{MB}$

Eindhoven, the Netherlands a.m.cohen@tue.nl

\section{JAN DRAISMA}

Department of Mathematics and Computing Science

Technische Universiteit Eindhoven P.O. Box 513, $5600 \mathrm{MB}$

Eindhoven, the Netherlands

j.draisma@tue.nl

\begin{abstract}
The action of an affine algebraic group $G$ on an algebraic variety $V$ can be differentiated to a representation of the Lie algebra $L(G)$ of $G$ by derivations on the sheaf of regular functions on $V$. Conversely, if one has a finite-dimensional Lie algebra $L$ and a homomorphism $\rho: L \rightarrow \operatorname{Der}_{K}(K[U])$ for an affine algebraic variety $U$, one may wonder whether it comes from an algebraic group action on $U$ or on a variety $V$ containing $U$ as an open subset. In this paper, we prove two results on this integration problem. First, if $L$ acts faithfully and locally finitely on $K[U]$, then it can be embedded in $L(G)$, for some affine algebraic group $G$ acting on $U$, in such a way that the representation of $L(G)$ corresponding to that action restricts to $\rho$ on $L$. In the second theorem, we assume from the start that $L=L(G)$ for some connected affine algebraic group $G$ and show that some technical but necessary conditions on $\rho$ allow us to integrate $\rho$ to an action of $G$ on an algebraic variety $V$ containing $U$ as an open dense subset. In the interesting cases where $L$ is nilpotent or semisimple, there is a natural choice for $G$, and our technical conditions take a more appealing form.
\end{abstract}

\section{Acknowledgments}

We thank Wilberd van der Kallen for pointing out Weil's theory of pre-transformation spaces to us, when that was exactly what we needed. We also thank Dmitri Zaitsev for his useful comment on an earlier version of this paper.

\section{Introduction}

Throughout this paper, $K$ denotes an algebraically closed field of characteristic 0 , and all algebraic varieties, algebraic groups and vector spaces are over $K$. If $a_{1}, \ldots, a_{k}$ are elements of a vector space $A$, then we denote by $\left\langle a_{1}, \ldots, a_{k}\right\rangle_{K}$ the subspace of $A$ spanned by $a_{1}, \ldots, a_{k}$. If $A$ is a $K$-algebra and $M$ is an $A$-bimodule, then a $K$-linear map $X$ from $A$ to $M$ satisfying

$$
X(a b)=X(a) b+a X(b) \text { for all } a, b \in A
$$

is called a $(K-)$ derivation of $A$ with values in $M$. We write $\operatorname{Der}_{K}(A, M)$ for the space of all such maps. Viewing $A$ as a bi-module over itself (on which left and right multiplication define the module structure), we abbreviate $\operatorname{Der}_{K}(A, A)$ to $\operatorname{Der}_{K}(A)$. This

Received. Accepted . 
space, whose elements are called $(K$-)derivations on $A$, is a Lie algebra with respect to the commutator.

Let $D^{(n)}$ be the Lie algebra of all $K$-derivations on the algebra $K\left[\left[x_{1}, \ldots, x_{n}\right]\right]$ of formal power series in the variables $x_{1}, \ldots, x_{n}$. Its elements are of the form

$$
\sum_{i=1}^{n} f_{i} \partial_{i}
$$

where the $f_{i}$ are elements of $K\left[\left[x_{1}, \ldots, x_{n}\right]\right]$, and $\partial_{i}$ denotes differentiation with respect to $x_{i}$. Let $D_{0}^{(n)}$ be the subalgebra of $D^{(n)}$ consisting of all derivations that leave the maximal ideal invariant. Note that $D_{0}^{(n)}$ has codimension $n$ in $D^{(n)}$. The Realization Theorem of Guillemin and Sternberg [7] states that any pair $(L, M)$, where $L$ is a Lie algebra and $M$ is a subalgebra of $L$ of codimension $n$, has a realization: a homomorphism $\phi: L \rightarrow D^{(n)}$ such that $\phi^{-1}\left(D_{0}^{(n)}\right)=M$. The formal power series occurring as coefficients of the $\partial_{i}$ in the image of $\phi$ are called the coefficients of the realization. The proof of the Realization Theorem presented in [1] is constructive in the sense that it allows for computation of these coefficients up to any desired degree. Moreover, in some special cases, one can prove that these coefficients are in fact polynomials, or polynomials and exponentials [5]. However, if $L$ is the Lie algebra of an affine algebraic group, and $M$ is the Lie algebra of a closed subgroup, the following construction is more natural.

Let $G$ be an affine algebraic group with unit $e$, and denote the stalk at $e$ of the sheaf of regular functions on open subsets of $G$ by $\mathcal{O}_{e}$. The Lie algebra of $G$, which coincides with $T_{e}(G)=\operatorname{Der}_{K}\left(\mathcal{O}_{e}, K\right)$ as a vector space, is denoted by $L(G)$. Let $V$ be an algebraic variety, and $\alpha: G \times V \rightarrow V$ a morphic action of $G$ on $V$, i.e., an action that is also a morphism of algebraic varieties. Then we can 'differentiate' $\alpha$ to a representation of $L(G)$ by derivations on $K[U]$, for any open affine subset $U$ of $V$. Assuming that $U$ is clear from the context, this representation is denoted by $X \mapsto-X *_{\alpha}$; its construction, and the presence of the minus sign, is explained in Subsection 2.4.

As a special case, take $V=G / H$, where $H$ is a closed subgroup of $G$. The group $G$ acts on $V$ by $\alpha\left(g_{1}, g_{2} H\right):=g_{1} g_{2} H$. Let $U$ be an affine open neighbourhood of $p:=e H$. Then $\left(X *_{\alpha} f\right)(p)=0$ for all $f \in K[U]$ if and only if $X \in L(H)$. Passing to the completion of the local ring $\mathcal{O}_{p}$ at $p$, we find a realization of the pair $(L(G), L(H))$ into $D^{(\operatorname{dim} V)}$, whose coefficients are algebraic functions. For example, if $G$ is connected and semisimple, and $H$ is parabolic, then $e H$ has an open neighbourhood in $G / H$ that is isomorphic to an affine space. Consequently, the pair $(L(G), L(H))$ has a realization with polynomial coefficients. For $G$ classical, this realization is computed explicitly in [11].

This paper deals with a converse of the above construction: given a finite-dimensional Lie algebra $L$ and a homomorphism $\rho: L \rightarrow \operatorname{Der}_{K}(K[U])$ for some affine algebraic variety $U$, can we find an affine algebraic group $G$, an algebraic variety $V$ containing $U$ as an open dense subset, an action $\alpha: G \times V \rightarrow V$, and an embedding $L \rightarrow L(G)$ such that $\rho$ is the restriction of the homomorphism $X \mapsto-X *_{\alpha}$ ?

The two main results of this paper answer this central question affirmatively for many interesting cases.

To formulate our first result, we introduce the notion of locally finite representations on a vector space $A$. A subset $E$ of $\operatorname{End}_{K}(A)$ is said to be locally finite, if each element 
of $A$ is contained in a finite-dimensional subspace of $A$ which is invariant under all elements of $E$. A representation $\rho: L \rightarrow \operatorname{End}_{K}(A)$ of an associative algebra or Lie algebra $L$ over $K$ is called locally finite if $\rho(L)$ is locally finite. In this case, $L$ is said to act locally finitely. A homomorphism $\rho: G \rightarrow \mathrm{GL}(A)$ from an algebraic group $G$ is called locally finite if $\rho(G)$ is locally finite, and in addition $\rho$ is a homomorphism $G \rightarrow \mathrm{GL}(M)$ of algebraic groups for each finite-dimensional $\rho(G)$-invariant subspace $M$ of $A$. In this case, $G$ is said to act locally finitely.

In our central question, if $G$ is to act on $U$ itself, the action of $L$ on $K[U]$ must be locally finite (see Proposition 7). Conversely, we have the following theorem for $\rho$ an inclusion.

Theorem 1. Let $U$ be an affine algebraic variety, and let $L$ be a locally finite Lie subalgebra of $\operatorname{Der}_{K}(K[U])$. Then there exist a linear algebraic group $G$, a morphic action $G \times U \rightarrow U$, and an embedding $L \rightarrow L(G)$ such that the representation $X \mapsto-X *_{\alpha}$ restricts to the identity on $L$.

Note that we do not require $L$ to be the Lie algebra of an algebraic group. Indeed, in Example 1 we shall see that $L$ need not coincide with $L(G)$.

As an example, let $U$ be the affine line, with coordinate $Y$. Then the derivation $\partial_{Y}$ acts locally finitely on $K[U]=K[Y]$, and so does the derivation $Y \partial_{Y}$. On the other hand, the derivation $Y^{2} \partial_{Y}$ does not act locally finitely. Theorem 1 can therefore be applied to $\left\langle\partial_{Y}, Y \partial_{Y}\right\rangle_{K}$, but not to $\left\langle\partial_{Y}, Y \partial_{Y}, Y^{2} \partial_{Y}\right\rangle_{K}$. However, any differential equation of the form

$$
Y^{\prime}(T)=\lambda+\mu Y(T)+\nu Y(T)^{2}, \quad Y(0)=Y_{0}
$$

with $\lambda, \mu, \nu \in K$ has a solution which is a rational expression in $Y_{0}, T$ and $\exp (\alpha T)$ for some $\alpha \in K$. This observation is a key to our results in the case where $\rho$ is not locally finite.

More formally, we introduce the exponential map. For simplicity, let us assume that $U$ be irreducible, so that $K[U]$ is an integral domain with field $K(U)$ of fractions. Let $T$ be a variable, and denote by $K[U][[T]]$ the algebra of formal power series in $T$ with coefficients from $K[U]$. For $f_{1}, \ldots, f_{k} \in K[U][[T]]$, we denote by $K(U)\left(f_{1}, \ldots, f_{k}\right)$ the subfield of the field of fractions of $K[U][[T]]$ generated by the $f_{i}$. For $\nabla \in \operatorname{Der}_{K}(K[U])$, we define the map $\exp (T \nabla)$ from $K[U]$ to $K[U][[T]]$ as follows.

$$
\exp (T \nabla) f=\sum_{n=0}^{\infty} \frac{T^{n}}{n !} \nabla^{n}(f), \quad f \in K[U] .
$$

Here, we only mention two consequences of our second main result (which is Theorem 10).

Theorem 2. Let $L$ be a nilpotent Lie algebra, $U$ an irreducible affine algebraic variety, $\rho: L \rightarrow \operatorname{Der}_{K}(K[U])$ a Lie algebra homomorphism, and $X_{1}, \ldots, X_{k}$ a basis of $L$ such that $\left\langle X_{i}, \ldots, X_{k}\right\rangle_{K}$ is an ideal in $L$ for all $i=1, \ldots, k$.

Suppose that $\rho$ satisfies

$$
\exp \left(T \rho\left(X_{i}\right)\right) K[U] \subseteq K(U)(T)
$$


for all $i$.

Then there exist a connected linear algebraic group $G$ having $L$ as its Lie algebra, an algebraic variety $V$ containing $U$ as an open dense subset, and a morphic action $\alpha$ : $G \times V \rightarrow V$ such that the corresponding representation $X \mapsto-X *_{\alpha}, L \rightarrow \operatorname{Der}_{K}(K[U])$ coincides with $\rho$.

Theorem 3. Let $L$ be a semi-simple Lie algebra of Lie rank $l, U$ an irreducible affine algebraic variety, and $\rho: L \rightarrow \operatorname{Der}_{K}(K[U])$ a Lie algebra homomorphism. Choose a Cartan subalgebra $H \subseteq L$, and let $\Phi$ be the root system with respect to $H$. Choose a fundamental system $\Pi \subseteq \Phi$, and a corresponding Chevalley basis

$$
\left\{X_{\gamma} \mid \gamma \in \Phi\right\} \cup\left\{H_{\gamma} \mid \gamma \in \Pi\right\} .
$$

Suppose that $\rho$ satisfies

$$
\exp \left(T \rho\left(X_{\gamma}\right)\right) K[U] \subseteq K(U)(T)
$$

for all $\gamma \in \Phi$, and

$$
\exp \left(T \rho\left(H_{\gamma}\right)\right) K[U] \subseteq K(U)(\exp T)
$$

for all $\gamma \in \Pi$.

Then there exist an algebraic variety $V$ containing $U$ as an open dense subset, and a morphic action $\alpha: G \times V \rightarrow V$ of the universal connected semi-simple algebraic group $G$ with Lie algebra $L$ such that the corresponding Lie algebra homomorphism $X \mapsto-X *_{\alpha}$ coincides with $\rho$.

Here, the 'universal' connected semi-simple algebraic group $G$ with Lie algebra $L$ is the unique such group with the property that every finite-dimensional representation of $L$ is the differential of a representation of $G$.

Technical conditions on the exponentials as appearing in these theorems are shown to be necessary in Lemma 9. For example, the vector field $Y^{3} \partial_{Y}$ on the affine line cannot originate from an action of the additive or the multiplicative group, because $\exp \left(T Y^{3} \partial_{Y}\right) Y=Y / \sqrt{1-2 T Y^{2}}$, which is not a rational expression in $Y, T$ and some exponentials $\exp \left(\lambda_{i} T\right)$. In Example 4, Theorem 3 is applied to $L=\left\langle\partial_{Y}, Y \partial_{Y}, Y^{2} \partial_{Y}\right\rangle_{K}$, the example discussed above.

This paper is organized as follows. Section 2 deals with standard facts on affine groups and their actions on varieties. In Section 3, Theorem 1 is proved, and Section 4 presents the proof of our second main theorem, from which Theorems 2 and 3 readily follow. Finally, Section 5 discusses some possible extensions of our results.

\section{Preliminaries}

In this section we introduce some notation, and we collect some facts on affine algebraic groups that will be used later on. All of them are based on [2].

\subsection{Locally Finite Transformations}

Let $A$ be a finite-dimensional vector space and let $Y \in \operatorname{End}_{K}(A)$. Then we write $Y_{s}$ and $Y_{n}$ for the semi-simple and the nilpotent part of $Y$. Let $\Gamma$ be the $\mathbb{Z}$-module generated by the eigenvalues of $Y_{s}$ on $A$. Decompose $A=\oplus_{\lambda} M_{\lambda}$, where $Y_{s} m=\lambda m$ for all $m \in M_{\lambda}$. For any $\mathbb{Z}$-module homomorphism $\phi: \Gamma \rightarrow K$, let $Y_{\phi} \in \operatorname{End}_{K}(A)$ be defined by $Y_{\phi} m=\phi(\lambda) m$ for all $m \in M_{\lambda}$. The collection of all such $Y_{\phi}$ is denoted by $S(Y)$. 
Now let $A$ be any vector space (not necessarily finite-dimensional). If $Y \in \operatorname{End}_{K}(A)$ is locally finite, the finite-dimensional $Y$-invariant subspaces of $A$ form an inductive system. If $N \subseteq M \subseteq A$ are two such subspaces, then $\left(\left.Y\right|_{M}\right)_{s}$ and $\left(\left.Y\right|_{M}\right)_{n}$ leave $N$ invariant, and restrict to $\left(\left.Y\right|_{N}\right)_{s}$ and $\left(\left.Y\right|_{N}\right)_{n}$, respectively. It follows that there are unique $Y_{s}, Y_{n} \in \operatorname{End}_{K}(A)$ such that $\left.\left(Y_{s}\right)\right|_{M}=\left(\left.Y\right|_{M}\right)_{s}$ and $\left.\left(Y_{n}\right)\right|_{M}=\left(\left.Y\right|_{M}\right)_{n}$ for all finite-dimensional $Y$-invariant subspaces $M$ of $A$. Furthermore, each element of $S\left(\left.Y\right|_{M}\right)$ leaves $N$ invariant, and restricts to an element of $S\left(\left.Y\right|_{N}\right)$; this restriction is surjective. We denote by $S(Y)$ the projective limit of the $S\left(\left.Y\right|_{M}\right)$; it projects surjectively onto each $S\left(\left.Y\right|_{M}\right)$.

\subsection{Localization}

If $B$ is a commutative algebra, and $J$ is an ideal in $B$, then we denote by $B_{\{J\}}$ the localization $B\left[(1+J)^{-1}\right]$. If $B=K[U]$ for some irreducible affine algebraic variety $U$, and $J$ is a radical ideal, then the elements of $B_{\{J\}} \subseteq K(U)$ are rational functions on $U$ that are defined everywhere on the zero set of $J$.

\subsection{Comorphisms}

If $\alpha$ is a morphism from an algebraic variety $V$ to an algebraic variety $W$, and $U$ is an open subset of $W$, then $\alpha$ induces a comorphism from the algebra of regular functions on $U$ to the algebra of regular functions on $\alpha^{-1}(U)$. We denote this comorphism by $\alpha^{0}$ if $U$ is clear from the context. If $W$ is affine, then $U$ is implicitly assumed to be all of $W$. By abuse of notation, we also write $\alpha^{0}$ for the induced comorphism of local rings $\mathcal{O}_{\alpha(p)} \rightarrow \mathcal{O}_{p}$, where $p \in V$, and for the comorphism $K(W) \rightarrow K(U)$ of rings of rational functions if $\alpha$ denotes a dominant rational map. This notation is taken from [2].

\subsection{Differentiation of Group Actions}

Let $G$ be an affine algebraic group with unit $e, V$ an algebraic variety, and $\alpha: G \times V \rightarrow V$ a morphic action of $G$ on $V$. Then we can 'differentiate' $\alpha$ to a representation of $L(G)=T_{e}(G)=\operatorname{Der}_{K}\left(\mathcal{O}_{e}, K\right)$ as follows. Let $U$ be an open subset of $V$. For $p \in U$, define the map $\alpha_{p}: G \rightarrow V$ by $g \mapsto \alpha(g, p)$. It maps $e$ to $p$, so we may view the comorphism $\alpha_{p}^{0}$ as a homomorphism $\mathcal{O}_{p} \rightarrow \mathcal{O}_{e}$. A function $f \in \mathcal{O}_{V}(U)$ defines an element of $\mathcal{O}_{p}$, to which $\alpha_{p}^{0}$ may be applied. Given $X \in L(G)$, the function $X *_{\alpha} f$ defined pointwise by

$$
\left(X *_{\alpha} f\right)(p):=\left(X \circ \alpha_{p}^{0}\right) f, \quad p \in U,
$$

is an element of $\mathcal{O}_{V}(U)$. The map $X *_{\alpha}: f \mapsto X *_{\alpha} f$ is a $K$-derivation on $\mathcal{O}_{V}(U)$, and the map $X \mapsto-X *_{\alpha}$ is a homomorphism $L(G) \rightarrow \operatorname{Der}_{K}\left(\mathcal{O}_{V}(U)\right)$ of Lie algebras.

In this way, $L(G)$ acts by derivations on the sheaf of regular functions on $V$. As $V$ may not have any non-constant regular functions at all, it makes sense to compute these derivations on $\mathcal{O}_{V}(U)$ for an affine open subset $U$ of $V$, so that $\mathcal{O}_{V}(U)$ equals the affine algebra $K[U]$. Let us assume for convenience that $G$ and $V$ are irreducible; then so are $U$ and $G \times U$. In this case, $\alpha^{0}$ sends $K[U]$ to $\mathcal{O}_{G \times V} \alpha^{-1}(U)$, an element of which defines an element of $\mathcal{O}_{G \times U}\left[(G \times U) \cap \alpha^{-1}(U)\right]$ by restriction. This algebra consists of fractions $a / b$ where $a, b \in K[G \times U]$ and $b$ vanishes nowhere on $(G \times U) \cap \alpha^{-1}(U)$. In particular, $b(e,$.$) vanishes nowhere on U$ and is therefore invertible in $K[U]$. After dividing both $a$ and $b$ by $b(e,$.$) , we have that b$ is an element of $1+J$, where $J$ is the ideal in $K[G \times U]$ defining $\{e\} \times U$. Thus, we can view $\alpha^{0}$ as a map $K[U] \rightarrow K[G \times U]_{\{J\}}$. The derivation 
$\nabla:=X \otimes I_{K[U]}: K[G] \otimes K[U] \rightarrow K[U]$ (where $I_{K[U]}$ denotes the identity map on $K[U]$ ) is extended to $(K[G] \otimes K[U])_{\{J\}}$ by

$$
\nabla\left(\frac{a}{b}\right)=\frac{\nabla(a) b(e, .)-a(e, .) \nabla(b)}{b(e, .)^{2}}
$$

for $a \in K[G \times U]$ and $b \in 1+J$. As $b(e,$.$) is the constant 1$ on $U$, the right-hand side is an element of $K[U]$. We have thus extended $X \otimes I_{K[U]}$ to a derivation

$$
(K[G] \otimes K[U])_{\{J\}} \rightarrow K[U],
$$

also denoted by $X \otimes I_{K[U]}$, and we may write

$$
X *_{\alpha}=\left(X \otimes I_{K[U]}\right) \circ \alpha^{0} .
$$

\subsection{The Associative Algebra $K[G]^{\vee}$}

The following construction is based on $[2, \S 3.19]$. Let $G$ be an affine algebraic group. Denote the multiplication by $\mu: G \times G \rightarrow G$, and the affine algebra by $K[G]$. For vector spaces $V$ and $W$, we define a $K$-bilinear pairing

$$
\begin{aligned}
& \operatorname{Hom}_{K}(K[G], V) \times \operatorname{Hom}_{K}(K[G], W) \rightarrow \operatorname{Hom}_{K}(K[G], V \otimes W), \\
& (X, Y) \mapsto X \cdot Y:=(X \otimes Y) \circ \mu^{0} .
\end{aligned}
$$

The multiplication $\cdot$ turns $K[G]^{\vee}=\operatorname{Hom}_{K}(K[G], K)$ into an associative algebra, and the map

$$
X \mapsto I \cdot X, \quad K[G]^{\vee} \rightarrow \operatorname{End}_{K}(K[G])
$$

is a monomorphism from $K[G]^{\vee}$ onto the $K$-algebra of elements in $\operatorname{End}_{K}(K[G])$ commuting with all left translations $\lambda_{g}$ for $g \in G$, which are defined by

$$
\left(\lambda_{g} f\right)(x):=f\left(g^{-1} x\right), \quad f \in K[G] .
$$

We shall write $f * X$ for the function $(I \cdot X) f$, and $* X$ for the map $f \mapsto f * X, K[G] \rightarrow$ $K[G]$. In particular, $X \mapsto * X$ is a linear isomorphism from the tangent space $L(G)=$ $T_{e}(G)$ onto the Lie algebra of elements of $\operatorname{Der}_{K}(K[G])$ commuting with all $\lambda_{g}$.

We recall the following well-known fact.

Proposition 4. The universal enveloping algebra $U(L(G))$ of $L(G)$ is isomorphic to the associative algebra with one generated by $L(G)$ in $K[G]^{\vee}$.

\subsection{Algebraicity of Lie Algebras}

We reformulate some results of Chevalley on algebraicity of subalgebras of $L(G)$, where $G$ is an affine algebraic group $[2, \S 7]$. For $M \subseteq L(G)$, we let $\mathcal{A}(M)$ be the intersection of all closed subgroups of $G$ whose Lie algebras contain $M$, and for $X \in L(G)$ we write $\mathcal{A}(X):=\mathcal{A}(\{X\})$.

Recall that $*\left(K[G]^{\vee}\right)$ is locally finite; in fact, the proof that this is the case is almost identical to the proof of Proposition 7. For $X \in L(G)$, both the semi-simple part and the nilpotent part of $* X$ are in $* L(G)$, and we denote their pre-images in $L(G)$ by 
$X_{s}$ and $X_{n}$, respectively. As $K[G]$ contains a finite-dimensional faithful $L(G)$-module that generates $K[G]$ as an algebra, the $\mathbb{Z}$-module $\Gamma_{X}$ of eigenvalues of $X_{s}$ in $K[G]$ is finitely generated. As $\Gamma_{X}$ is a torsion-free finitely generated Abelian group, it is free, and we may choose a basis $\lambda_{1}, \ldots, \lambda_{d}$ of $\Gamma_{X}$. For a variable $T$, consider the map $\exp (T X): K[G] \rightarrow K[[T]]$ defined by

$$
\exp (T X) f:=\sum_{n=0}^{\infty} \frac{T^{n}}{n !} X^{n}(f), \quad f \in K[G]
$$

where $X^{n}$ is viewed as an element of $K[G]^{\vee}$. Alternatively, we could write this formal power series as $(\exp (T(* X)) f)(e)$, where the exponential is the one defined in Section 1 . Clearly, $\exp (T X)$ is a homomorphism of $K$-algebras, whence an element of $G(K[[T]])$ $[2, \S 1.5]$. However, the following lemma shows that the image lies in a much smaller algebra.

Lemma 5. If $X_{n}=0$, then the map $\exp (T X)$ is a homomorphism

$$
K[G] \rightarrow K\left[\exp \left( \pm \lambda_{1} T\right), \ldots, \exp \left( \pm \lambda_{d} T\right)\right]
$$

whence the comorphism of a homomorphism $\gamma:\left(K^{*}\right)^{d} \rightarrow G$ of algebraic groups. If $X_{n} \neq 0$, then $\exp (T X)$ is a homomorphism

$$
K[G] \rightarrow K\left[T, \exp \left( \pm \lambda_{1} T\right), \ldots, \exp \left( \pm \lambda_{d} T\right)\right],
$$

whence the comorphism of a homomorphism $\gamma: K \times\left(K^{*}\right)^{d} \rightarrow G$ of algebraic groups. In either case, $\gamma$ is an algebraic group monomorphism onto $\mathcal{A}(X)$.

Proof. By [2, Proposition 1.11], we may assume that $G$ is a closed subgroup of $\mathrm{GL}_{n}$ for some $n$, and we may view $X$ as an element of $\mathfrak{g} l_{n}$. After a change of basis, $X_{s}=$ $\operatorname{diag}\left(\nu_{1}, \ldots, \nu_{n}\right)$, where $\nu_{1}, \ldots, \nu_{n}$ generate $\Gamma_{X}$. Now

$$
\exp \left(T X_{s}\right)=\operatorname{diag}\left(\exp \left(\nu_{1} T\right), \ldots, \exp \left(\nu_{n} T\right)\right),
$$

and we have

$$
\forall m \in \mathbb{Z}^{n}: \sum_{i=1}^{n} m_{i} \nu_{i}=0 \Rightarrow \prod_{i=1}^{n} \exp \left(\nu_{i} T\right)^{m_{i}}=1
$$

By $[2, \S 7.3], \mathcal{A}\left(X_{s}\right)$ consists precisely of those matrices $\operatorname{diag}\left(\xi_{1}, \ldots, \xi_{n}\right)$ with the property that

$$
\forall m \in \mathbb{Z}^{n}: \sum_{i=1}^{n} m_{i} \nu_{i}=0 \Rightarrow \prod_{i=1}^{n} \xi_{i}^{m_{i}}=1,
$$

so that the above implies

$$
\exp \left(T X_{s}\right) \in \mathcal{A}\left(X_{s}\right)\left(K\left[\exp \left( \pm \nu_{1} T\right), \ldots, \exp \left( \pm \nu_{n} T\right)\right]\right) .
$$

Now, any specialization

$$
K\left[\exp \left( \pm \nu_{1} T\right), \ldots, \exp \left( \pm \nu_{n} T\right)\right] \rightarrow K
$$


sends $\exp \left(T X_{s}\right)$ to an element of $\mathcal{A}\left(X_{s}\right)$. The algebra on the left-hand side is isomorphic to the algebra

$$
K\left[\exp \left( \pm \lambda_{1} T\right), \ldots, \exp \left( \pm \lambda_{d} T\right)\right],
$$

and as the $\lambda_{i}$ are linearly independent over $\mathbb{Q}$, the $\exp \left(\lambda_{i} T\right)$ are algebraically independent over $K$, and the latter is the affine algebra of $\left(K^{*}\right)^{d}$. We have thus constructed the algebraic group homomorphism $G_{m}^{d} \rightarrow \mathcal{A}\left(X_{s}\right)$, where $G_{m}$ denotes the multiplicative group of $K$. It is injective, as the $\nu_{i}$ generate the same $\mathbb{Z}$-module as the $\lambda_{i}$. As $d$ is also the dimension of $\mathcal{A}\left(X_{s}\right)$, and as $\mathcal{A}\left(X_{s}\right)$ is connected, the homomorphism is surjective onto $\mathcal{A}\left(X_{s}\right)$.

In $[2, \S 7.3]$, it is also proved that the homomorphism corresponding to the comorphism $\exp \left(T X_{n}\right): K[G] \rightarrow K[T]$ is a monomorphism from the additive group $G_{a}$ of $K$ onto $\mathcal{A}\left(X_{n}\right) \subseteq G$. The lemma now follows from $\exp (T X)=\exp \left(T X_{n}\right) \exp \left(T X_{s}\right)$ and $\mathcal{A}(X)=$ $\mathcal{A}\left(X_{n}\right) \times \mathcal{A}\left(X_{s}\right)$ (direct product).

Recall the notation $S(\cdot)$ from Subsection 2.1. For $X \in L(G)$ the set $S(* X)$ is a subset of $* L(G)$; we denote its pre-image in $L(G)$ by $S(X)$. Now $L(\mathcal{A}(X))$ is spanned by $X_{n}$ and $S(X)$. More generally, we have the following theorem of Chevalley $[2, \S 7.3$ and Corollary 7.7].

Theorem 6. Let $M$ be a subset of $L(G)$. Then $L(\mathcal{A}(M))$ is generated by the $X_{n}$ and $S(X)$ as $X$ varies over $M$.

Example 1 shows a subalgebra $L$ of $L(G)$ that is not equal to $L(\mathcal{A}(L))$.

An element $X \in L(G)$ is called algebraic if $L(\mathcal{A}(X))=\langle X\rangle_{K}$ is one-dimensional. In this case, $\mathcal{A}(X)$ is isomorphic to either $G_{a}$ or $G_{m}$, and if we denote the usual affine coordinate on the additive or multiplicative group by $Y$, then the differential of the homomorphism $G_{a} \rightarrow G$ (respectively $G_{m} \rightarrow G$ ) constructed above sends the basis vector $\left.\partial_{Y}\right|_{0}$ of $L\left(G_{a}\right)$ (respectively $\left.Y \partial_{Y}\right|_{1}$ of $L\left(G_{m}\right)$ ) to $X$. Algebraic elements of $L(G)$ play a role in the formulation of our second main result, Theorem 10.

\section{The Locally Finite Case}

In this section we prove Theorem 1 . Let $G$ be an affine algebraic group, $U$ an affine algebraic variety, and $\alpha: G \times U \rightarrow U$ a morphic action. Then the Lie algebra homomorphism $X \mapsto-X *_{\alpha}$ from Section 1 can be described more directly.

Proposition 7. For $X \in K[G]^{\vee}$, define the $K$-linear map $X *_{\alpha}: K[U] \rightarrow K[U]$ by

$$
X *_{\alpha}:=\left(X \otimes I_{K[U]}\right) \circ \alpha^{0} .
$$

Then $X \mapsto X *_{\alpha}$ is an anti-homomorphism of associative $K$-algebras. Moreover, its image $\left(K[G]^{\vee}\right) *_{\alpha}$ is locally finite.

Proof. The fact that $\alpha$ is an action can be expressed in terms of comorphisms by

$$
\left(\mu^{0} \otimes I_{K[U]}\right) \circ \alpha^{0}=\left(I_{K[G]} \otimes \alpha^{0}\right) \circ \alpha^{0} .
$$

Let $X, Y \in K[G]^{\vee}$, and compute

$$
\begin{aligned}
(X \cdot Y) *_{\alpha} & =\left(\left((X \otimes Y) \circ \mu^{0}\right) \otimes I_{K[U]}\right) \circ \alpha^{0} \\
& =\left(X \otimes Y \otimes I_{K[U]}\right) \circ\left(\mu^{0} \otimes I_{K[U]}\right) \circ \alpha^{0},
\end{aligned}
$$


which, by the above remark, equals

$$
\begin{aligned}
& \left(X \otimes Y \otimes I_{K[U]}\right) \circ\left(I_{K[G]} \otimes \alpha^{0}\right) \circ \alpha^{0} \\
& =\left(Y \otimes I_{K[U]}\right) \circ \alpha^{0} \circ\left(X \otimes I_{K[U]}\right) \circ \alpha^{0} \\
& =\left(Y *_{\alpha}\right) \circ\left(X *_{\alpha}\right) .
\end{aligned}
$$

This proves the first statement. Next, if $f \in K[U]$ and $\alpha^{0}=\sum_{i=1}^{k} a_{i} \otimes b_{i}$ with $a_{i} \in K[G]$ and $b_{i} \in K[U]$, then clearly $\left(K[G]^{\vee}\right) *_{\alpha} f \subseteq\left\langle b_{1}, \ldots, b_{k}\right\rangle_{K}$. This proves the second statement.

Note that the local finiteness of $\left(K[G]^{\vee}\right) *_{\mu}$ on $K[G]$ is a special case of this proposition. The proof that $*\left(K[G]^{\vee}\right)$ is locally finite, a fact that we used in Subsection 2.6, is very similar. Also note the subtle difference between the seemingly identical formulas (2) and (3). In the latter, $\alpha^{0}$ is a map $K[U] \rightarrow K[G \times U]$, whereas in the former, it is a map $K[U] \rightarrow K[G \times U]_{\{J\}}$.

As a consequence of Proposition 7, the representation $X \mapsto-X *_{\alpha}$ of $L(G)$ on $K[U]$ is locally finite, and so is the representation $G \rightarrow \operatorname{Aut}(K[U])$ defined by $g \mapsto \lambda_{g}$, where $\left(\lambda_{g} f\right)(p):=f\left(g^{-1} p\right)$. The latter follows because $\lambda_{g}=e_{g^{-1}} *_{\alpha}$, where $e_{g} \in K[G]^{\vee}$ denotes evaluation in $g$. In fact, $X \mapsto-X *_{\alpha}$ is the derivative at $e$ of the map $g \mapsto \lambda_{g}$. Conversely, we have the following theorem.

Theorem 8. Let $B$ be a finitely generated $K$-algebra (not necessarily commutative), and let $L \subseteq \operatorname{Der}_{K}(B)$ be a finite-dimensional Lie subalgebra acting locally finitely on $B$. Then there exist an affine algebraic group $G$, a faithful locally finite representation $\rho: G \rightarrow \operatorname{Aut}(B)$, and an embedding $\phi: L \rightarrow L(G)$ such that the derivative $d_{e} \rho$ of $\rho$ at $e$ satisfies $\left(d_{e} \rho\right) \circ \phi=\mathrm{id}$.

Proof. For any finite-dimensional $L$-invariant subspace $M$ of $B$, denote by $L_{M}$ the restriction of $L$ to $M$, and set $\tilde{L}_{M}:=L\left(\mathcal{A}\left(L_{M}\right)\right)$. The $\tilde{L}_{M}$ form an inverse system; let $\tilde{L}$ be its projective limit. By Theorem 6 and the remarks of Subsection 2.1, the projections $\tilde{L}_{M} \rightarrow \tilde{L}_{N}$ for $N \subseteq M$ are surjective. Also, the projections $\tilde{L} \rightarrow \tilde{L}_{M}$ are all surjective.

By Theorem 6, the space $\tilde{L}$ can be viewed as the Lie subalgebra of $\operatorname{End}_{K}(B)$ generated by the $X_{n}$ and $S(X)$ as $X$ varies over $L$. We claim that all of these are derivations of $B$. To verify this, it suffices to check Leibniz' rule on eigenvectors of $X_{s}$. To this end, let $a, b \in B$ be such that $X_{s} a=\lambda a$ and $X_{s} b=\mu b$. This is equivalent to

$$
(X-\lambda)^{k} a=(X-\mu)^{l} b=0
$$

for some $k, l \in \mathbb{N}$. From the identity

$$
(X-(\lambda+\mu))^{m}(a b)=\sum_{i=0}^{m}\left(\begin{array}{c}
m \\
i
\end{array}\right)(X-\lambda)^{i}(a)(X-\mu)^{m-i}(b)
$$

it follows that the left-hand side is zero for some $m \in \mathbb{N}$. Hence,

$$
X_{s}(a b)=(\lambda+\mu) a b=X_{s}(a) b+a X_{s}(b),
$$


and $X_{s}$ is a derivation, and so is $X_{n}=X-X_{s}$. Now let $\phi$ be a $\mathbb{Z}$-module homomorphism from the $\mathbb{Z}$-span of the eigenvalues of $X_{s}$ to $K$. Then the map $X_{\phi} \in S(X)$ satisfies

$$
\left(X_{\phi} a\right) b+a\left(X_{\phi} b\right)=(\phi(\lambda)+\phi(\mu)) a b=\phi(\lambda+\mu) a b=X_{\phi}(a b) .
$$

We have thus found that $\tilde{L}$ is generated by, and hence consists of, derivations. Let $M$ be a finite-dimensional $L$-submodule of $B$ that generates $B$ as an algebra. We have seen that the projection $\tilde{L} \rightarrow \tilde{L}_{M}$ is surjective, but as $\tilde{L}$ consists of derivations, which are determined by their values on $M$, it is also injective. Hence, $\tilde{L}_{M}$ acts on $B$ by derivations. Let $G \subseteq \mathrm{GL}(M)$ be $\mathcal{A}\left(L_{M}\right)$. It follows that $G$ acts locally finitely, and by automorphisms, on $B$; by construction, the corresponding action of $L(G)=\tilde{L}_{M}$ restricts to the identity on $L$.

Note that the construction of $G$ does not depend on the choice of $M$. The triple $(G, \rho, \phi)$ constructed in the proof has the property that $\mathcal{A}(\phi(L))=G$, and with this additional condition it is unique in the following sense: if $\left(G^{\prime}, \rho^{\prime}, \phi^{\prime}\right)$ is another such triple, then there exists an isomorphism $\psi: G \rightarrow G^{\prime}$ such that $\rho^{\prime} \circ \psi=\rho$ and $\left(d_{e} \psi\right) \circ \phi=$ $\phi^{\prime}$. Indeed, for any finite-dimensional $G^{\prime}$-invariant subspace $M$ of $B$ that generates $B$ as an algebra, $G^{\prime}$ must be isomorphic to $\mathcal{A}\left(L_{M}\right)$, just like $G$, and this defines the required isomorphism $\psi$.

Now the first main theorem follows almost directly.

Proof of Theorem 1. Apply Theorem 8 to $B=K[U]$ to find $G$, and its representation on $K[U]$. Let $M \subseteq K[U]$ be a finite-dimensional $G$-invariant subspace that generates $K[U]$ as an algebra. Then the surjective $G$-equivariant map from the symmetric algebra generated by $M$ onto $K[U]$ allows us to view $U$ as a closed $G$-invariant subset of the dual $M^{\vee}$. This gives the morphic action of $G$ on $U$, and it is straightforward to verify the required property.

Let us consider two examples where the embedding $L \rightarrow L(G)$ is not an isomorphism.

Example 1. Let $U=\operatorname{Spec}_{K} K[X, Y]$ be the affine plane, and let

$$
L=\left\langle\lambda_{1} X \partial_{X}+\lambda_{2} Y \partial_{Y}, \partial_{Y}, X \partial_{Y}, \ldots, X^{r} \partial_{Y}\right\rangle_{K},
$$

where $\lambda_{1}, \lambda_{2} \in K$ are linearly independent over $\mathbb{Q}$, and $r \in \mathbb{N}$. The Lie algebra $L$ acts locally finitely on $K[X, Y]$. Indeed, for $f \in K[X, Y]$, any element $g \in U(L(G)) f$ satisfies

$$
\operatorname{deg}_{X}(g) \leq \operatorname{deg}_{X}(f)+r \operatorname{deg}_{Y}(f), \text { and } \operatorname{deg}_{Y}(g) \leq \operatorname{deg}_{Y}(f) .
$$

Hence, Theorem 8 applies. Following its proof, we choose the $L$-invariant space $M=$ $\left\langle Y, 1, X, X^{2}, \ldots, X^{r}\right\rangle_{K}$, which generates $K[X, Y]$. Denoting by $L_{M}$ the restriction of $L$ to $M$, the proof of Theorem 1 shows that $\tilde{L}_{M}:=L\left(\mathcal{A}\left(L_{M}\right)\right)$ acts by derivations on $K[X, Y]$. With respect to the given basis of $M$, the derivation $\lambda_{1} X \partial_{X}+\lambda_{2} X \partial_{Y}$ has matrix

$$
\operatorname{diag}\left(\lambda_{2}, 0, \lambda_{1}, 2 \lambda_{1}, \ldots, r \lambda_{1}\right),
$$

whereas the elements $X^{i} \partial_{Y}$ of $L$ act nilpotently on $M$. Hence, $\tilde{L}_{M}$ is generated (and in fact spanned) by $L_{M}$ and the linear map with matrix

$$
\operatorname{diag}(1,0, \ldots, 0) .
$$


The image of $\tilde{L}_{M}$ in $\operatorname{Der}_{K}(K[X, Y])$ is spanned by $L$ and $X \partial_{X}$. The algebraic group $G$ is a semi-direct product $G_{m}^{2} \ltimes G_{a}^{r+1}$ acting by

$$
\left(t_{1}, t_{2}, a_{0}, \ldots, a_{r}\right)(x, y)=\left(t_{1} x, t_{2} y+\sum_{i=0}^{r} a_{i} x^{i}\right) .
$$

Example 2. Let $U=\operatorname{Spec}_{K}(K[X, Y])$ be the affine plane, and let $L$ be the onedimensional Lie algebra spanned by $\partial_{X}+Y \partial_{Y}$. Clearly, $L$ acts locally finitely on $K[X, Y]$; the group $G$ of Theorem 1 is $G_{a} \times G_{m}$ acting on $U$ by $(a, b)(x, y)=(x+a, b y)$, and the image of its Lie algebra in $\operatorname{Der}_{K}(K[U])$ equals $\left\langle\partial_{X}, Y \partial_{Y}\right\rangle_{K}$.

\section{Weil's Pre-Transformation Spaces}

This section is concerned with Theorem 10. The need for this theorem becomes clear from the following example.

Example 3. Let $\mathrm{SL}_{n+1}$ act on the projective $n$-space in the natural way. Then, after choosing suitable coordinates $X_{i}$ on an affine part $\mathbb{A}^{n} \subseteq \mathbb{P}^{n}$, the corresponding homomorphism $X \mapsto-X *_{\alpha}, \mathfrak{s l}_{n+1} \rightarrow \operatorname{Der}_{K}\left(K\left[\mathbb{A}^{n}\right]\right)$ has image

$$
\left\langle\left\{\partial_{i}, X_{i} \partial_{j}, X_{i} E\right\}_{i, j}\right\rangle_{K}
$$

where $E=\sum_{i} X_{i} \partial_{i}$. Clearly, this Lie algebra is not locally finite, so that we cannot apply Theorem 1 .

Let $G$ be a connected affine algebraic group acting on an irreducible algebraic variety $V$ by means of a morphic action $\alpha: G \times V \rightarrow V$, and let $U \subseteq V$ be an open affine subvariety. Recall the definition of the map $X \mapsto X *_{\alpha}, L(G) \rightarrow \operatorname{Der}_{K}(K[U])$ and the definition of the exponential map from Section 1 as well as the definition of $\Gamma_{X}$ from Subsection 2.6. Note that if $X \in L(G)$ is algebraic, then there are two possibilities: either $X$ is nilpotent and $\Gamma_{X}=0$, or $X$ is algebraic and $\Gamma_{X}$ has rank 1 .

Lemma 9. Let $X \in L(G)$ and let $\lambda_{1}, \ldots, \lambda_{d}$ be a basis for $\Gamma_{X}$. Then

$$
\exp \left(T\left(X *_{\alpha}\right)\right) K[U] \subseteq K[U]\left[T, S_{1}, \ldots, S_{d}\right]_{\{P\}}
$$

where $S_{i}=\exp \left(\lambda_{i} T\right)$, and $P$ is the ideal generated by $T, S_{1}-1, \ldots, S_{d}-1$. In particular, if $X$ is nilpotent, then

$$
\exp \left(T\left(X *_{\alpha}\right)\right) K[U] \subseteq K[U][T]_{\{(T)\}},
$$

and if $X$ is algebraic and semisimple, then

$$
\exp \left(T\left(X *_{\alpha}\right)\right) K[U] \subseteq K[U]\left[S_{1}\right]_{\left\{\left(S_{1}-1\right)\right\}}
$$

Proof. We claim that

$$
\left(X *_{\alpha}\right)^{n}=\left(X^{n} \otimes I_{K[U]}\right) \circ \alpha^{0} .
$$


where $X^{n}$ is evaluated in the associative algebra $K[G]^{\vee}$. To prove this, proceed by induction on $n$. For $n=1$ it is Equation (2); suppose that it holds for $n$, and compute

$$
\begin{aligned}
\left(X *_{\alpha}\right)^{n+1} & =\left(X \otimes I_{K[U]}\right) \circ \alpha^{0} \circ\left(X^{n} \otimes I_{K[U]}\right) \circ \alpha^{0} \\
& =\left(X \otimes I_{K[U]}\right) \circ\left(X^{n} \otimes I_{K[G]} \otimes I_{K[U]}\right) \circ\left(I_{K[G]} \otimes \alpha^{0}\right) \circ \alpha^{0} \\
& =\left(X \otimes I_{K[U]}\right) \circ\left(X^{n} \otimes I_{K[G]} \otimes I_{K[U]}\right) \circ\left(\mu^{0} \otimes I_{K[U]}\right) \circ \alpha^{0} \\
& =\left(\left(\left(X^{n} \otimes X\right) \circ \mu^{0}\right) \otimes I_{K[U]}\right) \circ \alpha^{0} \\
& =\left(X^{n+1} \otimes I_{K[U]}\right) \circ \alpha^{0} .
\end{aligned}
$$

In the first equality, we used the induction hypothesis, and in the third we used the fact that $\alpha$ is a morphic action. The last equality uses the multiplication in $K[G]^{\vee}$ as defined in Section 2. The other equalities follow from easy tensor product manipulations.

Using the above, we can calculate

$$
\begin{aligned}
\exp \left(T X *_{\alpha}\right) & =\sum_{n=0}^{\infty} \frac{T^{n}}{n !}\left(X^{n} \otimes I_{K[U]}\right) \circ \alpha^{0} \\
& =\left(\exp (T X) \otimes I_{K[U]}\right) \circ \alpha^{0}
\end{aligned}
$$

Now $\alpha^{0}$ is a map $K[U] \rightarrow(K[G] \otimes K[U])_{\{J\}}$, where $J$ is as in Section 1 , and $\exp (T X)$ maps $K[G]$ into $K\left[T, S_{1}^{ \pm 1}, \ldots, S_{d}^{ \pm 1}\right]$. Under $\exp (T X)$, the ideal $J$ is mapped into the ideal $P$. This concludes the proof.

Remark 1. The proof of Lemma 9 shows that $\exp \left(T X *_{\alpha}\right)$ can be viewed as the comorphism of the rational map $\mathcal{A}(X) \times U \rightarrow U$ defined by the restriction of $\alpha$.

Now suppose that we are given a homomorphism $L(G) \rightarrow \operatorname{Der}_{K}(K[U])$ for some affine algebraic variety $U$. Then the above lemma gives a necessary condition for this homomorphism to come from a group action on an algebraic variety $V$ containing $U$ as an open subset. In a sense, this condition is also sufficient. Let us state our main theorem in full detail.

Theorem 10. Let $G$ be a connected affine algebraic group and let $X_{1}, \ldots, X_{k}$ be a basis of $L(G)$ consisting of algebraic elements. Let $U$ be an irreducible affine algebraic variety, and $\rho: L(G) \rightarrow \operatorname{Der}_{K}(K[U])$ a homomorphism of Lie algebras.

Denote by $\Sigma$ the set of indices $i$ for which $X_{i}$ is semi-simple (in its action on $K[G]$ ), and let $\lambda_{i} \in K$ be such that $\Gamma_{X_{i}}=\mathbb{Z} \lambda_{i}$ for $i \in \Sigma$. Denote by $N$ the set of indices $i$ for which $X_{i}$ is nilpotent.

Assume that the product map

$$
\pi: \mathcal{A}\left(X_{1}\right) \times \ldots \times \mathcal{A}\left(X_{k}\right) \rightarrow G
$$

maps an open neighbourhood of $(e, \ldots, e)$ isomorphically onto an open neighbourhood of $e \in G$, and suppose that

$$
\exp \left(T \rho\left(X_{i}\right)\right) \in \begin{cases}K(U)(T) & \text { if } i \in N, \text { and } \\ K(U)\left(\exp \left(\lambda_{i} T\right)\right) & \text { if } i \in \Sigma .\end{cases}
$$


Then there exist an algebraic variety $V$ containing $U$ as an open dense subset, and a morphic action $\alpha: G \times V \rightarrow V$, such that the map $X \mapsto-X *_{\alpha}, L(G) \rightarrow \operatorname{Der}_{K}(K[U])$ coincides with $\rho$. Indeed, up to equivalence, there exists a unique such pair $(V, \alpha)$ with the additional property that $V \backslash U$ contains no G-orbit.

Crucial in the proof of this theorem are Weil's results on pre-transformation spaces $[10,12]$. Before stating the special case that we need, let us sketch the contents of Zaitsev's paper treating (and extending) these results. First, an algebraic pre-group $G$ is an algebraic variety equipped with a dominant rational map $\mu: G \times G \rightarrow G$ satisfying the axioms of 'generic associativity' and 'generic existence and uniqueness of left and right divisions'. Zaitsev proves that any algebraic pre-group $G$ has a unique regularization [12, Theorem 3.7 ], that is: an algebraic group $\tilde{G}$ with multiplication $\tilde{\mu}$ and a birational map $G \rightarrow \tilde{G}$ carrying $\mu$ over into $\tilde{\mu}$. A point of $G$ where this map is biregular is called a point of regularity of $G$; these points form an open dense subset of $G[12$, Lemma 3.9].

Next, if $G$ is an algebraic pregroup, then a pre-transformation $G$-space is an algebraic variety $U$ equipped with a dominant rational map $G \times U \rightarrow U,(g, p) \mapsto g p$ satisfying the axioms of 'generic associativity' and 'generic existence and uniqueness of left divisions'. A point $p_{0} \in U$ is called a point of regularity of $U$ if the map $p \mapsto g p$ is biregular at $p=p_{0}$ for generic $g \in G$. A regularization of $U$ consists of a pre-transformation $G$-space $V$ and a generically $G$-equivariant birational map $\psi: U \rightarrow V$ such that the following conditions are satisfied: $g \tilde{p}$ is defined whenever $g$ is a point of regularity of $G$ and $\tilde{p}$ is any point of $\tilde{U}$, and $\psi$ is biregular at any point of regularity of $U$. Zaitsev proves that any pre-transformation space $U$ has a regularization [12, Theorem 4.9]. There even exists a unique regularization $\tilde{U}$ of $U$ which is minimal in the sense that no proper open subset of $\tilde{U}$ is also a regularization of $U[12$, Theorem 4.12].

Note that, if $G$ is an algebraic group rather than just a pre-group, then $G$ acts morphically on the regularization $\tilde{U}$.

We will now formulate a special case of these results that we will use in the proof of Theorem 10. Recall that a rational map $\beta$ has a natural 'largest possible' domain; this domain is denoted by $\operatorname{dom}(\beta)$.

Lemma 11. Let $G$ be a connected algebraic group with multiplication $\mu: G \times G \rightarrow G$, $U$ an algebraic variety, and $\beta: G \times U \rightarrow U$ a dominant rational map such that

$$
\beta \circ\left(\operatorname{id}_{G} \times \beta\right)=\beta \circ\left(\mu \times \operatorname{id}_{U}\right)
$$

as dominant rational maps $G \times G \times U \rightarrow U$. Assume, moreover, that $\{e\} \times U \subseteq \operatorname{dom}(\beta)$, and that $\beta(e, p)=p$ for all $p \in U$.

Then there exist an algebraic variety $V$, an open immersion $\psi: U \rightarrow V$ with dense image, and a morphic action $\alpha: G \times V \rightarrow V$ such that $\alpha \circ\left(\operatorname{id}_{G} \times \psi\right)$ and $\beta$ define the same rational map. Indeed, up to equivalence, there exists a unique such triple $(V, \psi, \alpha)$ with the additional property that $V \backslash \psi(U)$ contains no G-orbit.

Proof. We show that $\beta$ makes $U$ into a pre-transformation $G$-space, in which every point is a point of regularity. First, generic associativity follows from the condition on $\beta$. Second, we must show generic existence and uniqueness of left divisions, i.e., that the rational map $(g, p) \mapsto(g, \beta(g, p))$ is in fact a birational map $G \times U \rightarrow G \times U$. Indeed, 
using generic associativity, and the fact that $\beta(e, p)=p$ for all $p \in U$, we find that $(g, p) \mapsto\left(g, \beta\left(g^{-1}, p\right)\right)$ is a rational map inverse to it.

Finally, let $p_{0} \in U$. Then the set $\Omega$ of $g \in G$ for which both $\left(g, p_{0}\right) \in \operatorname{dom}(\beta)$ and $\left(g^{-1},\left(\beta\left(g, p_{0}\right)\right)\right) \in \operatorname{dom}(\beta)$ is open, and non-empty as $e \in \Omega$. As $G$ is connected, $\Omega$ is dense in $G$. Let $g_{0} \in \Omega$, and consider the following rational maps $U \rightarrow U: p \mapsto \beta\left(g_{0}, p\right)$ and $p \mapsto \beta\left(g_{0}^{-1}, p\right)$. The first is defined at $p_{0}$ and the second at $\beta\left(g_{0}, p_{0}\right)$. Hence, both compositions are rational maps $U \rightarrow U$. Again, using generic associativity and the fact that $\beta(e, p)=p$ for all $p \in U$, we find that the two maps are each other's inverses. This shows that $p_{0}$ is a point of regularity. We may now apply Theorem 4.12 of [12], stating the existence and uniqueness of minimal a regularization of $U$, to find $V, \alpha$, and $\psi$. The proof of that theorem shows that $\psi$, which is a priori just a birational map $U \rightarrow V$, is an open immersion on the set of points of regularity, which is all of $U$. As $G$ is an algebraic group rather than just a pregroup, $\alpha$ is a morphic action of $G$ on $V$.

Proof of Theorem 10. Let $\epsilon_{i}=0$ or 1 if $i \in N$ or $i \in \Sigma$, respectively. By the property of $\pi$, the homomorphism

$$
\exp \left(T_{1} X_{1}\right) \cdot \ldots \cdot \exp \left(T_{k} X_{k}\right)
$$

identifies $K(G)$ with the field $K\left(S_{1}, \ldots, S_{k}\right)$, and $\mathcal{O}_{e}$ with the localization $K[S]_{M}:=$ $K\left[S_{1}, \ldots, S_{k}\right]_{M}$, where $S_{i}=T_{i}$ if $i \in N$ and $S_{i}=\exp \left(\lambda_{i} T_{i}\right)$ if $i \in \Sigma$, and $M$ is the (maximal) ideal generated by the elements $S_{i}-\epsilon_{i}$. The $K$-algebra $K[[T]]:=K\left[\left[T_{1}, \ldots, T_{k}\right]\right]$ can now be viewed as $\hat{\mathcal{O}}_{e}$, the completion of $K[S]_{M}$ with respect to the $M$-adic topology.

The co-multiplication

$$
\mu^{0}: \mathcal{O}_{e}=K[S]_{M} \rightarrow K\left[S^{\prime}, S^{\prime \prime}\right]_{M^{\prime} \otimes K\left[S^{\prime \prime}\right]+K\left[S^{\prime}\right] \otimes M^{\prime \prime}}=\mathcal{O}_{(e, e)}
$$

extends uniquely to a continuous homomorphism

$$
\mu^{0}: \hat{\mathcal{O}}_{e}=K[[T]] \rightarrow K\left[\left[T^{\prime}, T^{\prime \prime}\right]\right]=\hat{\mathcal{O}}_{(e, e)},
$$

and we have

$$
\begin{aligned}
\exp \left(\mu^{0}\left(T_{1}\right) X_{1}\right) \cdot \ldots \cdot \exp \left(\mu^{0}\left(T_{k}\right) X_{l}\right) & \\
& =\exp \left(T_{1}^{\prime} X_{1}\right) \cdot \ldots \cdot \exp \left(T_{k}^{\prime} X_{k}\right) \cdot \exp \left(T_{1}^{\prime \prime} X_{1}\right) \cdot \ldots \cdot \exp \left(T_{k}^{\prime \prime} X_{k}\right) .
\end{aligned}
$$

Similarly, the evaluation map $f \mapsto f(e), \mathcal{O}_{e} \rightarrow K$ extends to the continuous map $f \mapsto f(0), K[[T]] \rightarrow K$, where $K$ is given the discrete topology. Also, $X_{i} \in \operatorname{Der}_{K}\left(\mathcal{O}_{e}, K\right)$ extends uniquely to a continuous $K$-derivation $K[[T]] \rightarrow K$, where $K$ is given the structure of a $K[[t]]$-module defined by $f c=f(0) c$. This extension satisfies

$$
X_{i}\left(T_{j}\right)=\delta_{i, j} .
$$

Consider the map

$$
\beta^{0}:=\exp \left(-T_{k} \rho\left(X_{k}\right)\right) \cdot \ldots \cdot \exp \left(-T_{1} \rho\left(X_{1}\right)\right): K[U] \rightarrow K[U]\left[\left[T_{1}, \ldots, T_{k}\right]\right],
$$

where we implicitly extend each $\rho\left(X_{i}\right)$ linearly and continuously to formal power series with coefficients from $K[U]$. From the fact that the $\rho\left(X_{i}\right)$ are derivations, one finds 
that $\beta^{0}$ is a homomorphism. It is clearly injective, hence it extends to an injective homomorphism $K(U) \rightarrow K(U)\left(S_{1}, \ldots, S_{k}\right)$ by assumption. The latter field is identified with $K(G \times U)$ by the identification of $K(G)$ with $K\left(S_{1}, \ldots, S_{k}\right)$, and it follows that we may view $\beta^{0}$ as the comorphism of a dominant rational map $\beta: G \times U \rightarrow U$. We claim that the triple $(G, U, \beta)$ satisfies the conditions of Lemma 11.

To see this, denote by $P$ the ideal in $K[U]\left[S_{1}, \ldots, S_{k}\right]$ generated by the $S_{i}-\epsilon_{i}$, and let $f \in K[U]$. As $\beta^{0}(f)$ is a formal power series in the $T_{i}$, we find that, in the notation of Subsection 2.2,

$$
\beta^{0}(f) \in K[U]\left[S_{1}, \ldots, S_{k}\right]_{\{P\}} .
$$

We may identify the latter algebra with the algebra $K[G \times U]_{\{J\}}$ where $J$ is the radical ideal in $K[G \times U]$ defining $\{e\} \times U$. Hence, $\{e\} \times U \subseteq \operatorname{dom}\left(\beta^{0}(f)\right)$ for all $f \in K[U]$, which proves that $\{e\} \times U \subseteq \operatorname{dom}(\beta)$. Moreover, $\beta^{0}(f)(e, p)=f(p)$ for all $f \in K[U]$, from which it follows $\beta(e, p)=p$ for all $p \in U$.

Before proving generic associativity, we extend the map $-\rho$ to an anti-homomorphism $\tau$ from $U(L(G))$ into $\operatorname{End}_{K}(K[U])$, which can be done in a unique way. By Proposition 4 we may view $U(L(G))$ as the associative algebra with one generated by $L(G)$ in $K[G]^{\vee}$. We extend $\tau$ linearly and continuously to formal power series with coefficients from $U(L(G))$. Also, we extend the map $\mu^{0}: K\left[\left[T_{1}, \ldots T_{k}\right]\right] \rightarrow K\left[\left[T_{1}^{\prime}, \ldots, T_{k}^{\prime}, T_{1}^{\prime \prime}, \ldots, T_{k}^{\prime \prime}\right]\right]$ to a map

$$
U(L(G))\left[\left[T_{1}, \ldots, T_{k}\right]\right] \rightarrow U(L(G))\left[\left[T_{1}^{\prime}, \ldots, T_{k}^{\prime}, T_{1}^{\prime \prime}, \ldots, T_{k}^{\prime \prime}\right]\right]
$$

by

$$
\mu^{0}\left(\sum_{m \in \mathbb{N}^{k}} u_{m} T^{m}\right)=\sum_{m \in \mathbb{N}^{k}} u_{m} \mu^{0}\left(T^{m}\right) .
$$

Note that $\tau \circ \mu^{0}=\mu^{0} \circ \tau$. Indeed, $\tau$ acts only on $U(L(G))$ and $\mu^{0}$ only on the $T_{i}$.

We want to prove that

$$
\beta \circ\left(\operatorname{id}_{G} \times \beta\right)=\beta \circ\left(\mu \times \operatorname{id}_{U}\right)
$$

as dominant rational maps $G \times G \times U \rightarrow U$. This is equivalent to

$$
\left(I_{K[G]} \otimes \beta^{0}\right) \circ \beta^{0}=\left(\mu^{0} \otimes I_{K[U]}\right) \circ \beta^{0},
$$

for the comorphisms $K(U) \rightarrow K(G \times G \times U)$, and it suffices to prove this for the corresponding homomorphisms

$$
K[U] \rightarrow K[U]\left[\left[T_{1}^{\prime}, \ldots, T_{k}^{\prime}, T_{1}^{\prime \prime}, \ldots, T_{k}^{\prime \prime}\right]\right],
$$

where we use $T_{1}^{\prime}, \ldots, T_{k}^{\prime}$ for the generators of $\hat{\mathcal{O}}_{e}$ on the first copy of $G$, and $T_{1}^{\prime \prime}, \ldots, T_{k}^{\prime \prime}$ for those on the second copy. Compute

$$
\begin{aligned}
& \left(I_{K[G]} \otimes \beta^{0}\right) \circ \beta^{0} \\
& =\exp \left(-T_{k}^{\prime \prime} \rho\left(X_{k}\right)\right) \cdot \ldots \cdot \exp \left(-T_{1}^{\prime \prime} \rho\left(X_{1}\right)\right) \cdot \exp \left(-T_{k}^{\prime} \rho\left(X_{k}\right)\right) \cdot \ldots \cdot \exp \left(-T_{1}^{\prime} \rho\left(X_{1}\right)\right) \\
& =\tau\left(\exp \left(T_{1}^{\prime} X_{1}\right) \cdot \ldots \cdot \exp \left(T_{k}^{\prime} X_{k}\right) \exp \left(T_{1}^{\prime \prime} X_{1}\right) \cdot \ldots \cdot \exp \left(T_{k}^{\prime \prime} X_{k}\right)\right),
\end{aligned}
$$


to which we apply Equation (4), and find

$$
\begin{aligned}
& \tau\left(\exp \left(\mu^{0}\left(T_{1}\right) X_{1}\right) \cdot \ldots \cdot \exp \left(\mu^{0}\left(T_{k}\right) X_{k}\right)\right) \\
& =\tau\left(\mu^{0}\left(\exp \left(T_{1} X_{1}\right) \cdot \ldots \cdot \exp \left(T_{k} X_{k}\right)\right)\right) \\
& =\mu^{0}\left(\tau\left(\exp \left(T_{1} X_{1}\right) \cdot \ldots \cdot \exp \left(T_{k} X_{k}\right)\right)\right) \\
& =\mu^{0}\left(\exp \left(-T_{k} \rho\left(X_{k}\right)\right) \cdot \ldots \cdot \exp \left(-T_{1} \rho\left(X_{1}\right)\right)\right) \\
& =\left(\mu^{0} \otimes I_{K[U]}\right) \circ \beta^{0}
\end{aligned}
$$

as required.

Now that we have checked the conditions of Lemma 11, let $V$ and $\alpha: G \times V \rightarrow V$ be as in the conclusion of that lemma. For $f \in K[U]$ we have

$$
\begin{aligned}
X_{i} *_{\alpha} f & =\left(X_{i} \otimes I\right) \alpha^{0}(f) \\
& =\left(X_{i} \otimes I\right) \beta^{0}(f) \\
& =\left(X_{i} \otimes I\right)\left(\exp \left(-T_{k} \rho\left(X_{k}\right)\right) \cdot \ldots \cdot \exp \left(-T_{1} \rho\left(X_{1}\right)\right) f\right) \\
& =-\rho\left(X_{i}\right)(f) .
\end{aligned}
$$

In the last step we used that $X_{i}\left(T_{j}\right)=\delta_{i, j}$. This finishes the proof of the existence of $V$ and $\alpha$.

As for the uniqueness, suppose that $V$ and $\alpha$ satisfy the conclusions of the theorem. Then $\alpha$ defines a rational map $G \times U \rightarrow U$. From Remark 1, we find that this rational map coincides with $\beta$ defined above. Hence, the uniqueness of $(V, \alpha)$ follows from the uniqueness of a minimal regularization of $(U, \beta)$, see Lemma 11 .

The following lemma shows that the conditions on $G$ in Theorem 10 are not all that rare.

Lemma 12. Let $G$ be a connected affine algebraic group over $K$. Then $G$ has onedimensional closed connected subgroups $H_{1}, \ldots, H_{k}$ such that the product map $H_{1} \times$ $\ldots \times H_{k} \rightarrow G$ is an open immersion.

This fact is well known; see for example [2] and [6]. As we use the proof later, e.g. in the proof of Theorem 3, we give a brief sketch of it.

Proof. By a result of Mostow, the unipotent radical $R_{u}(G)$ of $G$ has a reductive Levi complement $G^{\prime}$, i.e., $G=G^{\prime} \ltimes R_{u}(G)[2, \S 11.22]$. Hence, it suffices to prove the proposition for $G$ reductive and for $G$ unipotent.

If $G$ is unipotent, then we can choose a basis $\left\{X_{i}\right\}_{i=1}^{k}$ of $L(G)$ such that $\left\langle X_{i}, \ldots, X_{k}\right\rangle_{K}$ is an ideal in $L(G)$ for all $i$, and the $H_{i}=\mathcal{A}\left(X_{i}\right) \cong G_{a}$ are subgroups as required.

If $G$ is reductive, choose a maximal torus $T$ of $G$, and a Borel subgroup $B^{+}$of $G$ containing $T$. Let $B^{-}$be the opposite Borel subgroup $[2, \S 14.1]$, and set $U^{ \pm}:=R_{u}\left(B^{ \pm}\right)$. Then it is known that the product map $U^{-} \times T \times U^{+} \rightarrow G$ is an open immersion; now $U^{-}$and $U^{+}$are dealt with by the unipotent case, and $T$ is isomorphic to $G_{m}^{d}$ for some $d$. 
Remark 2. It is not true that the product map $\mathcal{A}\left(X_{1}\right) \times \ldots \times \mathcal{A}\left(X_{k}\right) \rightarrow G$ is an open immersion for every basis $X_{1}, \ldots, X_{k}$ of $L(G)$ consisting of algebraic elements. Indeed, consider $G=G_{m}^{2}$ with Abelian Lie algebra $K^{2}$. The elements $X_{1}=(1,0), X_{2}=(1,2)$ are algebraic and form a basis of $L(G)$. We have

$$
\mathcal{A}\left(X_{1}\right)=\left\{(a, 1) \mid a \in K^{*}\right\} \text { and } \mathcal{A}\left(X_{2}\right)=\left\{\left(b, b^{2}\right) \mid b \in K^{*}\right\} .
$$

The product map $\mathcal{A}\left(X_{1}\right) \times \mathcal{A}\left(X_{2}\right) \rightarrow G$ is in fact a group homomorphism with kernel $\{((1,1),(1,1)),((-1,1),(-1,1))\}$.

Let us show how Theorems 2 and 3 follow from Theorem 10.

Proof of Theorem 2. By Harish-Chandra's refinement of Ado's theorem [8], $L$ has a faithful finite-dimensional representation $\phi: L \rightarrow \operatorname{End}_{K}(M)$ such that $L$ acts nilpotently on $M$. Let $G$ be the algebraic group $\mathcal{A}(\phi(L))$, where $\mathcal{A}$ is defined with respect to $\operatorname{GL}(M)$. By Theorem $6, L(G)=L$, and $G$ is easily seen to be unipotent. Let $H_{i}$ be the closed connected subgroup with $L\left(H_{i}\right)=K X_{i}$. The proof of Lemma 12 shows that the product map $H_{1} \times \ldots \times H_{k} \rightarrow G$ is an isomorphism of varieties. Hence, the conditions of Theorem 10 are fulfilled, and its conclusion finishes the proof.

Proof of Theorem 3. The proof of Lemma 12 shows that we can order the Chevalley basis in such a way that the product map from the product of the corresponding oneparameter subgroups into $G$ is an open immersion. In order to apply Theorem 10, it suffices to check that $\Gamma_{H_{i}}=\mathbb{Z}$ for all $i$. First, it is contained in $\mathbb{Z}$, as $H_{i}$ has only integer eigenvalues on any finite-dimensional $L$-module. Conversely, for $n \in \mathbb{Z}$, there exists a cyclic $L$-module $V$ on which $H_{i}$ has $n$ among its eigenvalues. As $G$ is universal, $V$ is also a $G$-module, and by [9, Satz II.2.4.1], $V$ is a submodule of $K[G]$. This proves that $\Gamma_{H_{i}}=\mathbb{Z}$. Application of Theorem 10 concludes the proof.

Example 4. Consider $G=\mathrm{SL}_{2}$. We identify the Lie algebra $L(G)$ with the vector space spanned by the matrices

$$
E=\left(\begin{array}{ll}
0 & 1 \\
0 & 0
\end{array}\right), H=\left(\begin{array}{cc}
1 & 0 \\
0 & -1
\end{array}\right), \text { and } F=\left(\begin{array}{ll}
0 & 0 \\
1 & 0
\end{array}\right),
$$

endowed with the usual Lie bracket for matrices. Here $E$ and $F$ are nilpotent, and $H$ is semisimple with $\Gamma_{H}=\mathbb{Z}$. The product map $\mathcal{A}(E) \times \mathcal{A}(H) \times \mathcal{A}(F) \rightarrow G$ is an open immersion by the proof of Lemma 12 .

Consider, for $U$, the affine line $\mathbb{A}^{1}$ with coordinate $Y$, and the homomorphism $\rho$ : $L(G) \rightarrow \operatorname{Der}_{K}(K[Y])$ defined by

$$
\rho(E)=-\partial_{Y}, \rho(H)=-2 Y \partial_{Y}, \text { and } \rho(F)=Y^{2} \partial_{Y} .
$$

It satisfies

$$
\begin{aligned}
\exp (T \rho(E))(Y) & =Y-T, \\
\exp (T \rho(H))(Y) & =\exp (-2 T) Y, \text { and } \\
\exp (T \rho(F))(Y) & =\frac{Y}{1-T Y}
\end{aligned}
$$


so that the conditions of Theorem 10 are fulfilled. It follows that there exists a unique algebraic variety $V$ containing $U=\mathbb{A}^{1}$ on which $G$ acts morphically, such that the corresponding Lie algebra representation equals $\rho$. Indeed, this variety $V$ is the projective line $\mathbb{P}^{1}$, on which $G$ acts by Möbius transformations.

Note that $\mathrm{PSL}_{2}$, on which $H$ has $\Gamma_{H}=2 \mathbb{Z}$, also acts on $\mathbb{P}^{1}$; this is reflected by the fact that $\exp (T \rho(H)) K[Y] \subseteq K(Y)(\exp (2 T))$, which is clearly a stronger statement than $\exp (T \rho(H)) K[Y] \subseteq K(Y)(\exp (T))$.

Note also that the Borel subalgebra $\langle E, H\rangle_{K}$ acts locally finitely on $K[Y]$, hence the corresponding Borel subgroup of $G$ acts on the affine line by Theorem 1.

Similarly, the vector fields realizing $\mathfrak{s} l_{n+1}$ in Example 3 can be used to recover the projective $n$-space from its affine part, as well as the action of $\mathrm{SL}_{n+1}$ on the former.

\section{Conclusion}

Although our two main results deal with different cases, Theorem 1 is more satisfactory than Theorem 10 in that it constructs the algebraic group from the Lie algebra. This raises the following question: let $L$ be a Lie algebra, $U$ an irreducible affine algebraic variety, and $\rho: L \rightarrow \operatorname{Der}(K[U])$ a Lie algebra homomorphism. Suppose that for all $X \in L$, there exist $\lambda_{1}, \ldots, \lambda_{d} \in K$ such that

$$
\exp (T \rho(X)) K[U] \subseteq K(U)\left(T, \exp \left(\lambda_{1} T\right), \ldots, \exp \left(\lambda_{d} T\right)\right)
$$

Do there exist an embedding $\phi$ of $L$ into the Lie algebra of an affine algebraic group $G$ and a morphic action $\alpha$ of $G$ on an algebraic variety $V$ containing $U$ as an open dense subset, such that

$$
\rho(X)=-\phi(X) *_{\alpha}
$$

for all $X \in L$ ?

The case where $L$ is one-dimensional is already interesting: suppose that $\nabla \in$ $\operatorname{Der}_{K}(K[U])$ satisfies

$$
\exp (T \nabla) K[U] \subseteq K(U)\left(T, \exp \left(\lambda_{1} T\right), \ldots, \exp \left(\lambda_{d} T\right)\right)
$$

where the $\lambda_{i}$ are independent over $\mathbb{Q}$. Are there mutually commuting derivations $\nabla_{0}, \nabla_{1}, \ldots, \nabla_{d} \in \operatorname{Der}_{K}(K[U])$ such that $\nabla=\nabla_{0}+\nabla_{1}+\ldots+\nabla_{d}$, and $\exp \left(T \nabla_{0}\right) K[U] \subseteq$ $K(U)(T)$ and $\exp \left(T \nabla_{i}\right) K[U] \subseteq K(U)\left(\exp \left(\lambda_{i} T\right)\right)$ for all $i$ ? The answer is yes, and the proof goes along the lines of the proof of Theorem 10: view $T, \exp \left(\lambda_{1} T_{1}\right), \ldots, \exp \left(\lambda_{d} T_{d}\right)$ as coordinates on $G:=G_{a} \times\left(G_{m}\right)^{d}$. Then $\exp (-T \nabla)$ is the comorphism of a rational map $\beta: G \times U \rightarrow U$, and one can show that the triple $(G, U, \beta)$ satisfies the conditions of Lemma 11. Let $V$ and $\alpha: G \times V \rightarrow V$ be as in the conclusion of that lemma. Then $\nabla=-\left(1, \lambda_{1}, \ldots, \lambda_{d}\right) *_{\alpha}$, and one can take $\nabla_{0}=-(1,0, \ldots, 0) *_{\alpha}$ and $\nabla_{i}=$ $-\left(0, \ldots, 0, \lambda_{i}, 0, \ldots, 0\right) *_{\alpha}$ for $i=1, \ldots, d$. Writing $\nabla_{n}=\nabla_{0}$ and $\nabla_{s}=\nabla_{1}+\ldots+\nabla_{d}$, we obtain a kind of Jordan decomposition of $\nabla$. It would be interesting to investigate whether such a decomposition is unique.

As an example, consider the derivation

$$
\nabla=\left(\lambda_{1} Y+Y^{2}\right) \partial_{Y}+\left(\lambda_{2} Z+Y Z\right) \partial_{Z}
$$


of $K[Y, Z]$, where $\lambda_{1}, \lambda_{2}$ are independent over $\mathbb{Q}$. It satisfies

$$
\begin{aligned}
\exp (T \nabla) Y & =\frac{\lambda_{1} \exp \left(\lambda_{1} T\right) Y}{\left(1-\exp \left(\lambda_{1} T\right)\right) Y+\lambda_{1}} \text { and } \\
\exp (T \nabla) Z & =\frac{\lambda_{1} \exp \left(\lambda_{2} T\right) Z}{\left(1-\exp \left(\lambda_{1} T\right)\right) Y+\lambda_{1}} .
\end{aligned}
$$

The right-hand sides are both in $K[Y, Z]\left(S_{1}, S_{2}\right)$, where $S_{i}=\exp \left(\lambda_{i} T\right)$ for $i=1,2$. If we view the (algebraically independent) $S_{i}$ as coordinates on $G_{m}^{2}$, the rational map $\beta$ is given by

$$
\beta\left(\left(s_{1}^{-1}, s_{2}^{-1}\right),(y, z)\right)=\left(\frac{\lambda_{1} s_{1} y}{\left(1-s_{1}\right) y+\lambda_{1}}, \frac{\lambda_{1} s_{2} z}{\left(1-s_{1}\right) y+\lambda_{1}}\right) .
$$

Differentiating the group action, we find that

$$
\begin{aligned}
& -(1,0) *_{\alpha}=\left(\frac{1}{\lambda_{1}} Y^{2}+Y\right) \partial_{Y}+\frac{1}{\lambda_{1}} Y Z \partial_{Z} \text { and } \\
& -(0,1) *_{\alpha}=Z \partial_{Z},
\end{aligned}
$$

so that indeed $\nabla=-\lambda_{1}(1,0) *_{\alpha}-\lambda_{2}(0,1) *_{\alpha}$.

In order to answer the question for higher-dimensional $L$, it seems that one should consider the Lie algebra $\tilde{L}$ generated by all vector fields $\nabla_{i}$ as $\nabla$ varies over $\rho(L)$, and prove that $\tilde{L}$ comes from an algebraic group, perhaps by constructing the latter from $\tilde{L}$ by means of the Campbell-Baker-Hausdorff formula.

Returning to realizations with nice coefficients (see Section 1), we let $L$ be a finitedimensional Lie algebra, and $M$ a subalgebra of $L$ of codimension $n$. Let $G$ be a connected affine algebraic group and $H$ a closed subgroup of $G$, also of codimension $n$. Assume that we have a homomorphism $\phi: L \rightarrow L(G)$ such that $\phi^{-1}(L(H))=M$. Now if we construct a realization $\psi: L(G) \rightarrow D^{(n)}$ as outlined in Section 1 , then $\psi \circ \phi$ is a realization of $(L, M)$. In particular, if $e H$ has an open neighbourhood isomorphic to $\mathbb{A}^{n}$ (either in $G / H$ or in some smooth $G$-equivariant compactification), then the pair $(L, M)$ has a realization with polynomial coefficients. This realization does not always seem to be obtainable by our algorithm based on Blattner's construction $([1,5])$. For example, in [5], it is proved that $(\mathfrak{k} \oplus \mathfrak{k}, \mathfrak{p})$, where $\mathfrak{k}$ is a simple Lie algebra and $\mathfrak{p}$ is the diagonal subalgebra of $\mathfrak{k} \oplus \mathfrak{k}$, has a realization with coefficients that are polynomials in the variables $x_{i}$ and some exponentials $\exp \left(\lambda x_{i}\right)$; this realization can be computed with our algorithm. However, as Michel Brion pointed out to us, it is known that the corresponding homogeneous space $(K \times K) / P$ - and in fact any spherical variety - has a smooth equivariant compactification that is covered by vector spaces $[3,4]$, so that the pair $(\mathfrak{k} \oplus \mathfrak{k}, \mathfrak{p})$ has a realization with polynomial coefficients only. It would be of interest to have computer algebra tools to compute such realizations explicitly; to do this by means of group theoretic methods, e.g. invariant theory, seems computationally very hard.

\section{References}

[1] Robert J. Blattner. Induced and produced representations of Lie algebras. Trans. Am. Math. Soc., 144:457-474, 1969. 
[2] Armand Borel. Linear Algebraic Groups. Springer-Verlag, New York, 1991.

[3] Michel Brion. Variétés sphériques. Notes of the S.M.F. session Opérations Hamiltoniennes et opérations de groupes algébriques, Grenoble 1997; available from

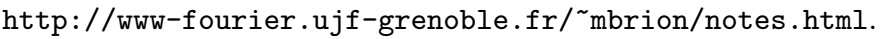

[4] Michel Brion, Domingo Luna, and Thierry Vust. Espaces homogènes sphériques. Invent. Math., 84:617-632, 1986.

[5] Jan Draisma. On a conjecture of Sophus Lie. To appear in the proceedings of the workshop Differential Equations and the Stokes Phenomenon, Groningen, The Netherlands, May 830, 2001.

[6] Alexander Grothendieck. Generalités sur les groupes algébriques affines. Groupes algébriques affines commutatifs. In Séminaire Claude Chevalley, volume 1, pages 4-14-14, Paris, 1958. École Normale Supérieure.

[7] Victor W. Guillemin and Shlomo Sternberg. An algebraic model of transitive differential geometry. Bull. Am. Math. Soc., 70:16-47, 1964.

[8] Harish-Chandra. Faithful representations of Lie algebras. Ann. Math., 50(1), 1949.

[9] Hanspeter Kraft. Geometrische Methoden in der Invariantentheorie. Friedr. Vieweg \& Sohn, Braunschweig/Wiesbaden, 1984.

[10] André Weil. On algebraic groups of transformations. Am. J. Math., 77:355-391, 1955.

[11] Pavel Winternitz and Louis Michel. Families of transitive primitive maximal simple Lie subalgebras of $\operatorname{diff}_{n}$. CRM Proc. Lect. Notes., 11:451-479, (1997).

[12] Dmitri Zaitsev. Regularization of birational group operations in the sense of Weil. J. Lie Theory, 5:207-224, 1995. 